\title{
Distribución de las Arterias Coronarias en el Rinoceronte Blanco (Ceratotherium simum)
}

\author{
Distribution of Coronary Arteries in the White Rhinoceros (Ceratotherium simum) \\ William Pérez \& Martin Lima
}

PÉREZ, W. \& LIMA, M. Distribución de las arterias coronarias en el rinoceronte blanco (Ceratotherium simum). Int. J. Morphol., 28(3):811-814, 2010.

RESUMEN: Nosotros disecamos un corazón de rinoceronte blanco con el objetivo de dar una descripción anatómica de la distribución de sus arterias coronarias. Se registró la longitud y el calibre de las ramas principales. Las arterias coronarias tenían el mismo calibre en su origen y la arteria coronaria derecha emitía la rama interventricular subsinusal, el rinoceronte correspondía al patrón de coronaria derecha. La arteria coronaria izquierda emitida en el seno izquierdo de la aorta se dividía en tres ramas (trifurcación), la rama interventricular paraconal, la rama circunfleja y la rama diagonal.

PALABRAS CLAVE: Anatomía animal; Anatomía cardiaca; Animales silvestres; Rhinocerotidae; Vasos coronarios.

\section{INTRODUCCIÓN}

Las dos especies africanas de rinoceronte son el rinoceronte negro (Diceros bicornis) y el rinoceronte blanco (Ceratotherium simum). La Anatomía cardiaca del rinoceronte negro fue descrita por Matoussi (1998). También fue descrito el corazón de Rhinoceros unicornis, el rinoceronte de un cuerno de la India (Bhattacharya et al., 1987), pero esta descripción es muy general. En ambos trabajos los autores disecaron sólo un animal.

En lo que respecta a la distribución de las arterias coronarias hay cientos de publicaciones en la literatura zoológica y veterinaria. En los libros de texto clásicos de Getty (1975) y Barone (1996) se encuentran bien detalladas las distribuciones correspondientes a los mamíferos domésticos.

Nosotros no encontramos ninguna descripción anatómica de las arterias coronarias del rinoceronte blanco (Ceratotherium simum). Por lo tanto aprovechamos la oportunidad de la disección de un corazón de rinoceronte para describir las arterias coronarias con el objetivo de aumentar el conocimiento anatómico existente en esta especie.
MATERIAL Y MÉTODO

El corazón obtenido de un rinoceronte blanco necropsiado en un zoológico local fue disecado luego de fijación en una solución de formaldehído al 5\%. El peso corporal del animal no pudo ser obtenido. La distribución de los vasos sanguíneos arteriales fue disecada y fotografiada con una cámara digital Nikon D 9O. El diámetro interno de las principales arterias fue determinado con el uso de un calibre metálico. Los términos se utilizarón de acuerdo al International Committee on Veterinary Gross Anatomical Nomenclature (2005).

\section{RESULTADOS}

La aorta emitida en el centro de la base del corazón tenía un diámetro interno de $6.0 \mathrm{~cm}$ en su parte inicial luego de los senos aórticos y el espesor de su pared era de $1,5 \mathrm{~cm}$ (Fig. 1). El tronco pulmonar tenía un calibre $5,5 \mathrm{~cm}$ y un espesor de pared de $0,8 \mathrm{~cm}$. La arteria coronaria izquierda emitida en el seno izquierdo de la aorta se dividía en tres ramas (trifurcación), la rama interventricular paraconal, la rama circunfleja y la rama diagonal (Fig 2).

Área de Anatomía, Facultad de Veterinaria, Universidad de la República, Montevideo, Uruguay. 


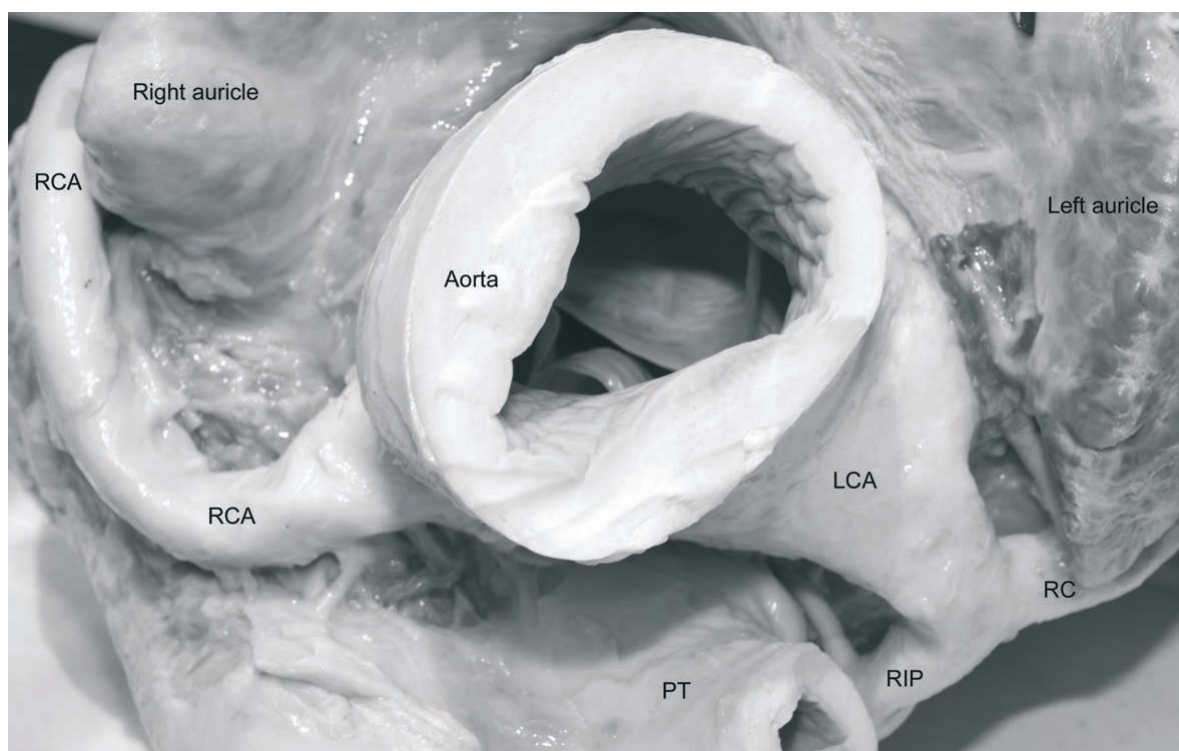

Fig. 1. Vista dorsal de la aorta en el origen de las arterias coronarias. RCA. Arteria coronaria derecha; LCA. Arteria coronaria izquierda; RC. Rama circunfleja. RIP. Rama interventricular paraconal.

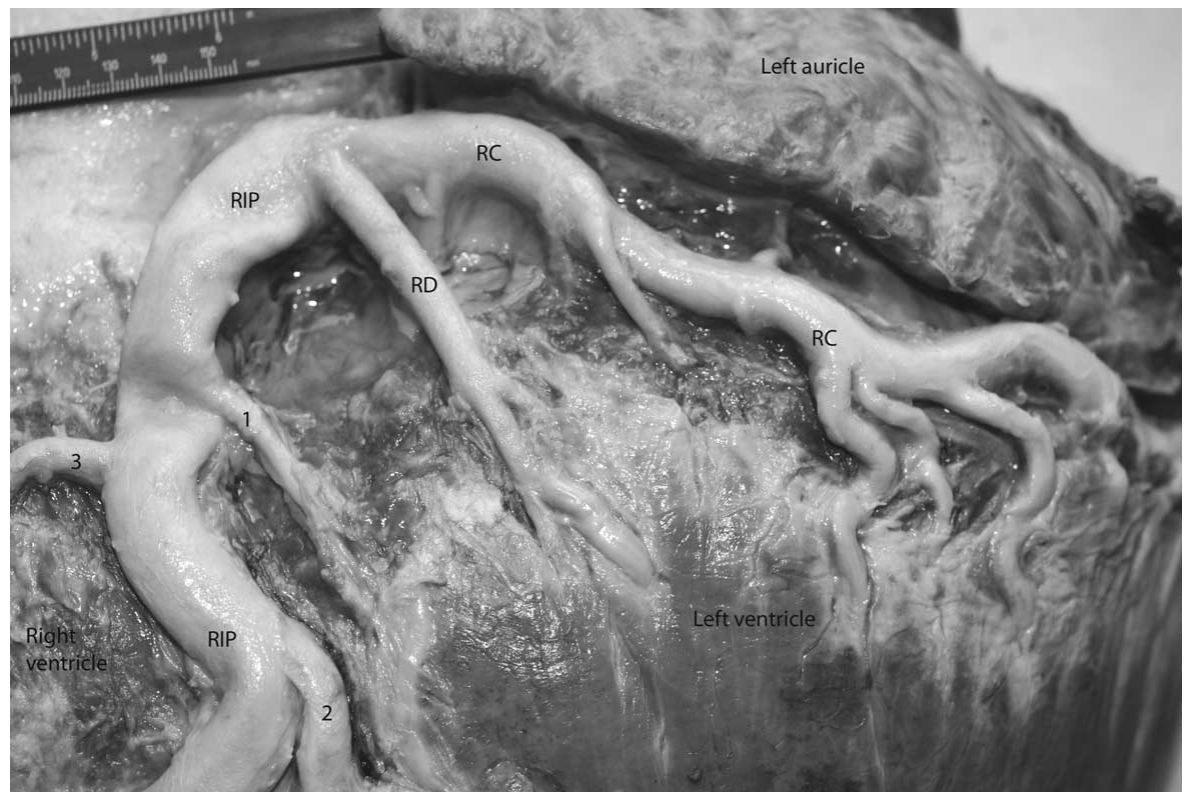

Fig. 2. Cara auricular del corazón, distribución de la arteria coronaria izquierda. RC. Rama circunfleja; RIP. Rama interventricular paraconal; RD. Rama diagonal. 1 y 2; Ramas al ventrículo izquierdo de la rama interventricular paraconal; 3. Rama al ventrículo derecho de la rama interventricular paraconal.

La arteria coronaria izquierda tenía un calibre de $1,7 \mathrm{~cm}$ y $4,0 \mathrm{~cm}$ de longitud (Figs. 1 y 2). La rama interventricular paraconal tenía el mismo calibre $(1,7 \mathrm{~cm})$ y una longitud de $34,0 \mathrm{~cm}$. (Fig. 2). Esta arteria emitía cranealmente hacia el corazón derecho diez ramas, la tercera de ellas luego de un trayecto de $3,5 \mathrm{~cm}$ se bifurcaba en dos ramas menores. Esta tercera rama era la rama del cono arterioso. Las dos primeras ramas iban al tronco pulmonar. Las diez ramas tenían cada una (de dorsal a ventral) una longitud de $4,0 \mathrm{~cm}$, $3,0 \mathrm{~cm}, 3,5 \mathrm{~cm}, 2,0 \mathrm{~cm}, 5,0 \mathrm{~cm}$, $2,1 \mathrm{~cm}, 1,3 \mathrm{~cm}, 1,5 \mathrm{~cm}, 1,0 \mathrm{~cm}$ y $5,0 \mathrm{~cm}$. La distancia de emisión entre el origen de la arteria coronaria izquierda y la primera rama derecha de la paraconal era de $2,0 \mathrm{~cm}$ y entre la primera y la segunda de $1,4 \mathrm{~cm}$, la segunda y la tercera estaban separadas por $2.8 \mathrm{~cm}$, la tercera y la cuarta por $6,7 \mathrm{~cm}$, la cuarta y la quinta por $0,4 \mathrm{~cm}$, la quinta y la sexta por $7,2 \mathrm{~cm}$, la sexta y la séptima por $0,9 \mathrm{~cm}$, la séptima y la octava por $0,4 \mathrm{~cm}$, la octava y la novena por $1,1 \mathrm{~cm}$ y la novena y la décima por $3,2 \mathrm{~cm}$. Las tres primeras ramas y la quinta eran las más voluminosas de las ramas derechas de la paraconal. El calibre de las ramas derechas era inferior al de las ramas izquierdas.

Hacia caudal, hacia el ventrículo izquierdo, la rama interventricular paraconal emitió 7 arterias. Estas ramas tenían cada una (de dorsal a ventral) una longitud de $6,0 \mathrm{~cm}, 10,0 \mathrm{~cm}, 5,0$ $\mathrm{cm}, 1,7 \mathrm{~cm}, 5,3 \mathrm{~cm}, 4,5 \mathrm{~cm}$ y 5,0 $\mathrm{cm}$. La distancia de emisión entre la diagonal y la primera de estas ramas era de $4,5 \mathrm{~cm}$, la primera y la segunda estaban separadas por $5,0 \mathrm{~cm}$, la segunda y la tercera por $3,0 \mathrm{~cm}$, la tercera y la cuarta por $5,0 \mathrm{~cm}$, la cuarta y la quinta por $3,0 \mathrm{~cm}$, la quinta y la sexta por $2,0 \mathrm{~cm}$ y la sexta y la última estaban separadas por 2,0 $\mathrm{cm}$. El calibre respectivo de estas siete ramas era de $1,0 \mathrm{~mm}, 4,0$ $\mathrm{mm}, 3 \mathrm{~mm}, 1 \mathrm{~mm}, 2 \mathrm{~mm}, 2 \mathrm{~mm}$ y $1 \mathrm{~mm}$. La segunda rama era la más voluminosa de las siete ramas de la rama interventricular paraconal.

Luego de la emisión de estas ramas la rama interventricular paraconal cruzó el borde ventricular derecho y descendió 
hacia el ápex ventralmente a la rama interventricular subsinusal.

La rama circunfleja (Fig. 2) tenía un calibre en su origen de $1,1 \mathrm{~cm}$ y se reducía gradualmente de calibre terminando a unos $\mathrm{cm}$ del surco interventricular paraconal. En su trayecto emitía 10 arterias que se distribuían hacia el ventrículo izquierdo y al borde ventricular izquierdo. La longitud de estas ramas era respectivamente de $3,0 \mathrm{~cm}, 2,2 \mathrm{~cm}$, $3,4 \mathrm{~cm}, 1,3 \mathrm{~cm}, 6,0 \mathrm{~cm}, 5,0 \mathrm{~cm}, 8,0 \mathrm{~cm}, 3,0 \mathrm{~cm}, 5,5 \mathrm{~cm}, 7,5$ $\mathrm{cm}$. El calibre de cada una era de $1,0 \mathrm{~mm}, 1,0 \mathrm{~mm}, 2,0 \mathrm{~mm}$, $1,0 \mathrm{~mm}, 3,0 \mathrm{~mm}, 2 \mathrm{~mm}, 2 \mathrm{~mm}, 1,0 \mathrm{~mm}, 1,0 \mathrm{~mm}$ y $2,0 \mathrm{~mm}$. Hacia dorsal la rama circunfleja emitió dos ramas mayores que se dirigían hacia el aatrio izquierdo, ambas tenían una longitud de $3,5 \mathrm{~cm}$ y un calibre de $1,0 \mathrm{~mm}$. La tercera rama de la arteria coronaria izquierda o rama diagonal (Fig. 2) tenía una longitud total de $10,0 \mathrm{~cm}$ y un calibre de $4,0 \mathrm{~mm}$ en su parte inicial. Esta arteria se distribuía exclusivamente al ventrículo izquierdo.

La arteria coronaria derecha (Fig. 1) que procedía del seno derecho de la aorta tenía el mismo calibre que su homóloga izquierda $(1,7 \mathrm{~cm})$, una longitud total de $33,0 \mathrm{~cm}$ y se continuaba por la rama interventricular subsinusal. Esta última rama tenía una longitud total de $30,0 \mathrm{~cm}$ y un calibre de $1,2 \mathrm{~cm}$. La rama interventricular subsinusal emitió 6 pequeñas arterias hacia cada ventrículo y terminó gradualmente antes de llegar al ápex cerca del pasaje de la rama interventricular paraconal.

La arteria coronaria derecha dio 4 finas ramas dorsales hacia el atrio derecho y ventralmente dio nacimiento a 9 ramas que se distribuían a las partes dorsales del ventrículo derecho. La longitud de estas ramas era respectivamente de $8,0 \mathrm{~cm}, 7,0 \mathrm{~cm}, 9,0 \mathrm{~cm}, 9,0 \mathrm{~cm}, 8,5 \mathrm{~cm}, 9 \mathrm{~cm}, 3,0 \mathrm{~cm}, 3,0$ $\mathrm{cm}$ y $5,0 \mathrm{~cm}$.

El septum interventricular estaba irrigado por varias ramas que procedían mayormente de las ramas interventriculares paraconal y subsinusal. En ninguna parte del corazón vimos anastomosis entre las ramas de las arterias coronarias.

\section{DISCUSIÓN}

En este trabajo, que hasta donde sabemos es la primera descripción de las arterias coronarias en el rinoceronte blanco, se disecaron las ramas de ambas arterias coronarias y se aportaron datos adicionales del calibre de las mismas, longitud y distancia entre la emisión de las ramas menores. Matoussi en su estudio del corazón de un rinoceronte negro registró datos de la longitud, calibre y distribución de los vasos sanguíneos principales.

En nuestro estudio e igual que en el rinoceronte negro las dos arterias coronarias tenían el mismo calibre. A diferencia del rinoceronte negro la arteria coronaria izquierda se dividía en tres ramas. La rama diagonal no se mencionó en el rinoceronte negro (Matoussi). La rama interventricular paraconal emitía siete ramas ventriculares izquierdas en nuestro corazón y seis en el rinoceronte negro.

La arteria coronaria derecha era más larga en el rinoceronte blanco que en el negro. Está arteria es la llamada circunfleja derecha en el trabajo de Matoussi. La rama interventricular subsinusal también era más larga en el rinoceronte blanco.

Los animales domésticos pueden se divididos en dos grupos acorde al patrón de distribución de ambas arterias coronarias. En el grupo A, la rama interventricular subsinusal es una rama de la arteria coronaria izquierda. Este tipo referido como tipo izquierdo se presenta en el bovino, ovino, caprino, canino y felino como indican Barone \& Colin, Christensen (1962), Hoffmann (1975), Ghoshal (1975 b,d). En la jirafa predomina la arteria coronaria izquierda (Pérez et al., 2008a). Lo mismo pasa en el delfin Pontoporia blainvillei (Pérez \& Lima, 2006).

En el grupo B, la rama interventricular subsinusal es rama de la arteria coronaria derecha. Este tipo es referido como tipo derecho y se adjudica al caballo y cerdo (Christensen; Hoffmann; Ghoshal, 1975a, c) al búfalo de agua de Egipto (George et al., 1969). A este grupo pertenecen el camello dromedario descrito en tres trabajos (Kanan, 1971; Ghazi \& Tadjalli, 1993; Taha \& Abel-Magied, 1996), y el camello bactriano (Yuan et al., 2009). En Arctocephalus australis la rama interventricular subsinusal procede en la mayoría de los animales de la arteria coronaria derecha (Pérez et al., 2008b).

Debido a que ambas arterias coronarias son iguales en calibre y similares en distribución en el rinoceronte blanco (nuestro estudio) y en el rinoceronte negro (Matoussi), estos animales son del grupo B, con la rama interventricular subsinusal emitida por la arteria coronaria derecha. Este patrón que también está presente en el equino (Hoffmann; Ghoshal, 1975a) parece ser característico de los perisodáctilos.

Este artículo se ha dedicado únicamente a la anatomía macroscópica de las arterias coronarias del rinoceronte blanco. Claramente se requieren estudios adicionales de un mayor número de animales para entender la anatomía cardíaca de este gran animal. 
PÉREZ, W. \& LIMA, M. Distribution of coronary arteries in the white rhinoceros (Ceratotherium simum). Int. J. Morphol., 28(3):811-814, 2010.

SUMMARY: We dissected the heart of white rhino in order to give an anatomical description of the distribution of coronary arteries. We recorded the length and caliber of the main branches. The coronary arteries were the same caliber at its origin and the right coronary artery emitted subsinusal interventricular branch, rhinoceros corresponded to the pattern of the right coronary artery. The left coronary artery is released into the left breast of the aorta was divided into three branches (trifurcation) paraconal interventricular branch, circumflex branch and diagonal branch.

KEY WORDS: Animal anatomy; Heart anatomy; Wild animals; Rhinocerotidae; Coronary vessels.

\section{REFERENCIAS BIBLIOGRÁFICAS}

Barone, R. Anatomie comparée des mammifères domestiques. Angiologie. Éditions Vigot, Paris, 1996. V. 5.

Barone, R. \& Colin, A. Les arteries du coeur chez les Ruminants domestiques. Rev. Med. Vet., 102:172-81, 1951.

Bhattacharya, M.; Baishya, G.; Chakraborty, A. \& Baruah, G. K. Gross Anatomy of the Heart of the Indian One Horned Rhinoceros (Rhinoceros unicornis). J. Zoo Anim. Med., 18 (23):105-8, 1987.

George, A. N.; Berge, R.; Moustafa, M. S. \& El-Gaffary, M. The coronary vessels in the Egyptian water buffalo (Bod Bubalus hubalis L.). Zbl. Vet. Med., 16:894-905, 1969.

Getty, R. Sisson and Grossman's Anatomy of the Domestic Animals. $5^{\text {th }}$ Edition. Philadelphia, London, Toronto, W. B. Saunders Company, 1975.

Ghazi, S. R. \& Tadjalli. M. Coronary arterial anatomy of the onehumped camel (Camelus dromedarius). Vet. Res. Commun., 17:163-70, 1993.

Ghoshal, N. G. Equine heart and arteries. In: Sisson and Grossman's Anatomy of The Domestic Animals. $5^{\text {th }}$ edition. Getty, R. (ed.). Philadelphia, London, Toronto, W. B. Saunders Company, 1975a. pp.554-618.

Ghoshal, N. G. Ruminant heart and arteries. In: Sisson and Grossman's Anatomy of The Domestic Animals. $5^{\text {th }}$ edition. Getty, R. (ed.). Philadelphia, London, Toronto, W. B. Saunders Company, 1975b. pp.960-1023.

Ghoshal, N. G. Porcine heart and arteries. In: Sisson and Grossman's Anatomy of The Domestic Animals. $5^{\text {th }}$ edition. Getty, R. (ed.). Philadelphia, London, Toronto, W. B. Saunders Company, 1975c. pp.1306-42.

Ghosal, N. G. Carnivore heart and arteries. In: Sisson and Grossman's Anatomy of The Domestic Animals. $5^{\text {th }}$ edition. Getty, R. (ed.). Philadelphia, London, Toronto, W. B. Saunders Company, 1975d. pp.1598-651.

Christensen, G. C. The blood supply to the interventricular septum of the heart - a comparative study. Am. J. Vet. Res., 23:869-74, 1962.
Hoffmann, V. Blood-vessel supply of the equine heart, also a comparative study of the topography of coronary vessels in domestic mammals (carnivores, swine and ruminants). Anat. Anz., 137(1-2):79-109, 1975.

International Committee on Veterinary Gross Anatomical Nomenclature (I.C.V.G.A.N.) Nomina Anatomica Veterinaria, 5th ed. 2005. http://www.wava-amav.org/Downloads/ nav_2005.pdf.

Kanan, C. V. Observations on the pattern and distribution of the coronary blood vessels of the camel (Camelus dromedarius). Acta Morphol. Neerl. Scand., 8(4):321-32, 1971.

Matoussi, A. Le coeur du rhinoceros bicorne d'Afrique (Diceros bicornis): Elements d'anatomie. Revue de Medecine Veterinaire, 149(5):407-16, 1998.

Pérez, W. \& Lima, M. Cardiac anatomy of the Pontoporia blainvillei. Int. J. Morphol., 24(3):351-6, 2006.

Pérez, W.; Lima, M.; Pedrana, G. \& Cirillo, F. Heart anatomy of Giraffa camelopardalis rothschildi: a case report. Veterinarni Medicina, 53(3):165-8, 2008a.

Pérez, W.; Katz, H. \& Lima, M. Gross heart anatomy of Arctocephalus australis (Zimmerman, 1783). Anat. Sci. Int., 83(1):6-10, 2008b.

Taha, A. A. \& Abel-Magied, E. M. The coronary arteries of the dromedary camel (Camelus dromedarius). Anat. Histol. Embryol., 25(4):295-9, 1996.

Yuan, G.; Ma, J.; Ye, W.; Bai, Z. \& Wang, J. Macroanatomy of coronary arteries in Bactrian camel (Camelus bactrianus). Vet. Res. Commun., 33(4):367-77, 2009.

Dirección para correspondencia:

Prof. William Pérez

Área de Anatomía, Facultad de Veterinaria

Universidad de la República

Lasplaces 1620, 11600 Montevideo

URUGUAY

Email: vetanat@gmail.com

Recibido : 25-12-2009

Aceptado: 12-03-2010 